\title{
Prävalenz von Asthma bei Kindern nimmt zu
}

\author{
Ist es eher die Atemluft oder mehr die Ernährung, die \\ bei Kindern die Entwicklung eines Asthma bronchia- \\ le fördert? Dieser Frage gingen Experten beim ERS- \\ Kongress in London nach.
}

Laut WHO gilt die Luftverschmutzung als eine von zehn Hautursachen von Atemwegserkrankungen. Wie es bei Kindern um die Assoziation zwischen Schadstoffbelastung der Atemluft und der Entwicklung der Lungenfunktion sowie der Prävalenz von allergischem und nicht allergischem Asthma bestellt ist, wurde in vielen Studien untersucht. Kinder sind für Schadstoffe in der Luft besonders anfällig, weil sie häufiger durch den Mund atmen, im Verhältnis zur Körpergröße eine schnellere Ventilationsrate und kleinere Atemwege haben und die Filterfunktion bei Nasenatmung ineffektiver ist. Ebenso spielen das sozioökonomische Umfeld der Familie, Passivrauchen, Atopie und genetische Variablen eine Rolle.

Sind Kinder im ersten Lebensjahr einer starken verkehrsbedingten Luftverschmutzung ausgesetzt, lassen sich im Schulkindalter im Serum erhöhte Werte für inflammatorische Zytokine wie Interleukin 6, IL-10, IL-13 oder TNF nachweisen. Auf Basis der Daten der schwedischen BAMSE-Studie mit über 4.000 Kindern bis zum Alter von 12 Jahren konnte eine Assoziation zwischen Luftverschmutzung durch Verkehr (Traffic-related air Pollution, TRAP) im ersten Lebensjahr und Asthma und Giemen in den ersten 12 Lebensjahren demonstriert werden (Gruzieva O et al., Epidemiology 2013, 24(1):54-61). Der stärkste Zusammenhang zeigte sich dabei zum nicht allergischen Asthma, fasste Dr. Olena Gruzieva vom Institut für Umweltmedizin am Karolinska-Institut, Stockholm, zusammen. TRAP war assoziiert mit erhöhten IL-10-Spiegeln bei Kindern mit Asthma und bei solchen, deren Mütter in der Schwangerschaft oder während der frühen Kindheit geraucht hatten.

Insgesamt bestehe eine zunehmende Evidenz für die Verursachung von Asthma durch Luftverschmutzung. Luftschadstoffe wie Feinstaub können sowohl das sich entwickelnde Immunsystem (z.B. T-Zell-Differenzierung) und das Lungenwachstum beeinflussen. Epigenetische Faktoren sind am Zusammenhang zwischen Umwelt und Genexpression beteiligt. Kritische Zeiten sind dabei insbesondere pränatale und frühkindliche Lebensabschnitte.

\section{Einfluss der Ernährung beginnt im Mutterleib}

In puncto Ernährung konnte ein Zusammenhang zwischen der Ernährung der Mutter in der Schwangerschaft und der kindlichen Entwicklung von Atemwegserkrankungen festgestellt werden, erläuterte Prof. Graham Devereux von der Universität Aberdeen. Als Einflussfaktoren werden drei Hypothesen diskutiert:

- Antioxidantien-Hypothese

- Lipid-Hypothese

- Vitamin-D-Hypothese

Wie Devereux zusammenfasste, könnten veränderte Ernährungsgewohnheiten, etwa ein Mangel an Antioxidatien durch geringeren Verzehr von Früchten und Gemüse, und eine Ver- änderte Zusammensetzung von Nahrungsmitteln bzgl. PUFA (mehrfach ungesättigte Fettsäuren), z.B. die Zunahme von n6-PUFA (in Margarine und Pflanzenöl) oder die Abnahme von n-3-PUFA (in fettem Fisch) in der Nahrung zu dem beobachteten Anstieg von Asthma und Atopien beitragen. Diskutiert wird auch ein Zusammenhang mit dem weit verbreiteten VitaminD-Mangel und dem Vitamin-E-Gehalt der Nahrung.

Studien haben gezeigt, dass Veränderungen in der Ernährung schwangerer Frauen in puncto PUFA und Vitamin E und D relevante Effekte haben. Möglicherweise wird damit das foetale Immunsystem und die Entwicklung der Atemwegsepithelien beeinflusst. Aktualisierte Ernährungsempfehlungen für Schwangere könnten möglicherweise zur Prävention von Asthma beitragen. Interventionen sollten jedoch nicht als Supplementierung erfolgen, sondern in Form von natürlichen Nahrungsbestandteilen einen synergistischen Effekt entfalten.

Dagmar Jäger-Becker

Quelle: Symposium „Childhood asthma causation“, ERS-Kongress, London, 3. -7.9 .2016$

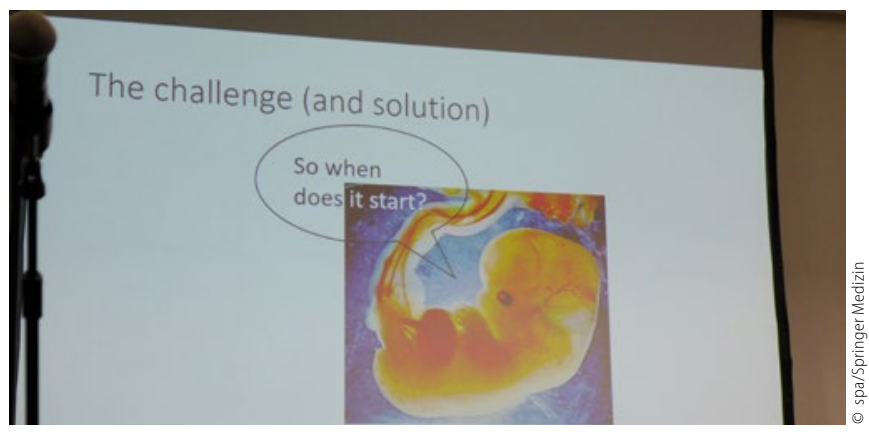

Feinstaub kann das Wachstum von Immunsystem und Lungen beeinflussen, auch epigenetische Faktoren sind am Asthma beteiligt.

\section{Gen-Umwelt-Interaktion}

\section{Stillen schützt vor Asthma}

Umweltfaktoren können das genetische Risiko für Asthma, das auf Chromosom 17 (17q21) lokalisiert ist, bekanntlich modifizieren. Kinder mit genetischen Varianten in 17q21 zeigen eine höhere Wahrscheinlichkeit für die Entwicklung von Giemen. Eine Studie mit 368 Kindern konnte jetzt erstmals nachweisen, dass Stillen dieses Risiko im ersten Lebensjahr reduzieren kann, erklärte Dr. Olga Gorlanova vom Universitäts-Kinderspital beider Basel (UKBB).

Wurden die Kinder gestillt, hatten die Träger des Asthma-Risiko-Genotyps ein um $27 \%$ geringeres relatives Risiko, respiratorische Symptome zu entwickeln. Ohne Stillen zeigten die Risiko-Gen-Träger dagegen einen Trend zur Entwicklung von Symptomen.

Diese Untersuchung vertieft den Einblick in die Gen-Umwelt-Interaktion bei der Entwicklung von Asthma.

Quelle: International Congress 2016 der European Respiratory Society ERS, London, 3.-7. September 2016 